\title{
Dense minors in graphs of large girth
}

Reinhard Diestel \& Christof Rempel

\begin{abstract}
We show that a graph of girth greater than $6 \log k+3$ and minimum degree at least 3 has a minor of minimum degree greater than $k$. This is best possible up to a factor of at most 9/4. As a corollary, every graph of girth at least $6 \log r+3 \log \log r+c$ and minimum degree at least 3 has a $K_{r}$ minor.
\end{abstract}

\section{Introduction}

Thomassen [9] proved that, in graphs of minimum degree at least 3 , sufficiently high girth forces a minor of any given minimum degree:

Theorem. (Thomassen 1983)

For any integer $k$, every graph $G$ of girth $g(G) \geqslant 4 k-3$ and $\delta(G) \geqslant 3$ has a minor $H$ with $\delta(H) \geqslant k$.

Our aim in this note is to reduce the upper bound for the required girth to the correct order of magnitude:

Theorem 1. For any integer $k$, every graph $G$ of girth $g(G)>6 \log k+3$ and $\delta(G) \geqslant 3$ has a minor $H$ with $\delta(H)>k$.

The best lower bound implied by known examples is $\frac{8}{3} \log k-c$, but we note that existing conjectures about cubic graphs of large girth would raise this to about $4 \log k$.

Since an average degree of at least $c r \sqrt{\log r}$ forces a $K_{r}$ minor $[5,10]$, Theorem 1 has the following consequence:

Corollary 2. There exists a constant $c \in \mathbb{R}$ such that every graph $G$ of girth $g(G) \geqslant 6 \log r+3 \log \log r+c$ and $\delta(G) \geqslant 3$ has a $K_{r}$ minor.

Asymptotically, Thomason [11] showed that a $K_{r}$ minor is forced by an average degree of $(d+o(1)) r \sqrt{\log r}$, where $d=0.53131 \ldots$ is an explicit constant that is best possible. This means that, for large enough $r$, Corollary 2 holds with $c=-2.4742$.

We adopt the notation of [4]. All our logarithms are binary, all graphs considered finite, and $0 \in \mathbb{N}$. 


\section{A lower bound}

Minimum-order cubic graphs of girth at least some given integer $g$ are called $g$-cages and have been studied in some detail (see [1] for an overview). Their exact order is known for $g \leqslant 12$. The best more general upper bound for the order of $g$-cages is due to Biggs \& Hoare [2] and Weiss [12]:

Lemma 2.1. There is a constant $c^{*}>0$ such that for infinitely many integers $g$ there exists a cubic graph of girth at least $g$ and order at most $c^{*} 2^{3 g / 4}$.

Now suppose that a graph $G$ as in Lemma 2.1 has a minor of minimum degree $k$ (say). Then $G$ has at least $k+1$ branch sets, each of which sends out at least $k$ edges and hence contains at least $k-2$ vertices (since $G$ is cubic). Therefore

$$
(k+1)(k-2) \leqslant|G| \leqslant c^{*} 2^{3 g / 4},
$$

giving $g \geqslant \frac{8}{3} \log k-c$ for a suitable constant $c$. Choosing $k=k(g) \in \mathbb{N}$ maximal with this last inequality, we can thus deduce from Lemma 2.1 the following counterpart to Theorem 1:

Proposition 2.2. There is a constant $c \in \mathbb{R}$ such that for infinitely many $k \in \mathbb{N}$ there exist cubic graphs of girth at least $\frac{8}{3} \log k-c$ that have no minor $H$ with $\delta(H)>k$.

Any improvement on the bound in Lemma 2.1 will result in a corresponding improvement to Proposition 2.2. It has been conjectured (see [3] or [8]) that $g$-cages exist on as few as about $2^{g / 2}$ vertices. This would increase our lower girth bound to $4 \log k-c$.

\section{The upper bound}

In this section we prove Theorem 1. Following Mader [7], we start from the observation that in a graph $G$ of girth $g(G)>2 d+1$ and $\delta(G) \geqslant 3$ the $d$ ball around a vertex $x$ is a tree $T_{x}$ sending at least $\left|T_{x}\right|-2$ edges to the rest of $G$. Our main effort will go into proving that, depending on our lower bound for $g(G)$, not too many of these edges can go to the same tree $T_{y}$. Then partitioning $V(G)$ into such trees and contracting these will give us a minor of large minimum degree.

Given a tree $T$ with root $r$ and vertices $t, t^{\prime} \in T$, we say that $t^{\prime}$ lies above $t$ in $T$ (and $t$ below $t^{\prime}$ ) if $t \leqslant t^{\prime}$ in the tree-order on $V(T)$ associated with $r$, ie. if $t$ separates $t^{\prime}$ from $r$ in $T$. Any neighbour of $t$ above it is a successor of $t$ in $T$, its unique neighbour below is its predecessor. For $i \in \mathbb{N}$ we write $L_{T}^{i}$ for the set of leaves (maximal elements) of $T$ at distance $i$ from $r$.

Given a graph $G$, a vertex $x \in G$, and $d \in \mathbb{N}$, let us write $V_{G, x}^{d}$ for the set of vertices of $G$ at distance exactly $d$ from $x$. We need the following easy lemma: 
Lemma 3.1. Let $T$ be a tree with root $r$ in which no vertex has exactly one successor, and let $d \in \mathbb{N}$. Then $\sum_{i \geqslant d} 2^{d-i}\left|L_{T}^{i}\right| \geqslant\left|V_{T, r}^{d}\right|$.

We are now ready to prove our main result, which we restate:

Theorem 1. For any integer $k$, every graph $G$ of girth $g(G)>6 \log k+3$ and $\delta(G) \geqslant 3$ has a minor $H$ with $\delta(H)>k$.

Proof. Put $\lfloor\log k\rfloor=: d$. Let $X$ be a maximal set of vertices such that $d(x, y)>2 d$ for all distinct $x, y \in X$. Beginning with $T_{x}^{0}:=\{x\}$, let us define trees $T_{x}^{i}$ rooted at $x$, for all $x \in X$ and $i=0, \ldots, 2 d$. Assume that for some $i$ the $T_{x}^{i}$ have been defined and partition the set of vertices of $G$ at distance at most $i$ from $X$. We then add each vertex $v$ at distance $i+1$ from $X$ to one $T_{x}^{i}$ to which it is adjacent, thereby obtaining a similar set of disjoint trees $T_{x}^{i+1}$. By the choice of $X$, the trees $T_{x}:=T_{x}^{2 d}$ partition the entire vertex set of $G$, and

$T_{x}$ contains all the vertices of $G$ at distance at most $d$ from $x$.

As $g(G)>4 d+1$, the $T_{x}$ are induced subgraphs in $G$. Finally, we have

$$
d(w, y) \leqslant d(v, x)+1 \text { whenever } v w \in E(G) \text { with } v \in T_{x} \text { and } w \in T_{y},
$$

as otherwise $w$ would have been added to $T_{x}$ after $v$ rather than to $T_{y}$.

Let us use Lemma 3.1 to estimate the number of edges leaving a tree $T_{x}$. For all $i \in \mathbb{N}$ let

$$
E_{x}^{i}:=\left\{v w \in E(G) \mid v \in T_{x}, w \in G-T_{x}, d(v, x)=i\right\}
$$

Let $T_{x}^{\prime}$ denote the subgraph of $G$ induced by $T_{x}$ and all its neighbours in $G$. As $g(G)>4 d+3, T_{x}^{\prime}$ is again a tree. Every vertex $v \in T_{x}$ has degree $d_{G}(v) \geqslant 3$ in $T_{x}^{\prime}$, while all the vertices of $T_{x}^{\prime}-T_{x}$ are leaves in $T_{x}^{\prime}$. As $\left|E_{x}^{i}\right|=\left|L_{T_{x}^{\prime}}^{i+1}\right|$ for all $i$, and $\left|L_{T_{x}^{\prime}}^{d}\right|=0$ by (1), Lemma 3.1 yields

$$
\sum_{i \geqslant d} 2^{d-i-1}\left|E_{x}^{i}\right|=\sum_{i \geqslant d} 2^{d-i-1}\left|L_{T_{x}^{\prime}}^{i+1}\right|=\sum_{i \geqslant d} 2^{d-i}\left|L_{T_{x}^{\prime}}^{i}\right| \geqslant\left|V_{T_{x}^{\prime}, x}^{d}\right|=\left|V_{G, x}^{d}\right| .
$$

Multiplying by $2^{d+1}$ and setting $V_{x}^{d}:=V_{G, x}^{d}$ we obtain

$$
\sum_{i \geqslant d} 2^{2 d-i}\left|E_{x}^{i}\right| \geqslant 2^{d+1}\left|V_{x}^{d}\right|
$$

Every edge in $E_{x}^{i}$ joins $T_{x}$ to a tree $T_{y}$ distinct from $T_{x}$. This defines a partition of $E_{x}^{i}$ into sets $A_{x, y}^{i}(y \in X \backslash\{x\})$. Then the above inequality can be rewritten as

$$
2^{d+1}\left|V_{x}^{d}\right| \leqslant \sum_{y} \sum_{i \geqslant d} 2^{2 d-i}\left|A_{x, y}^{i}\right|
$$


where the first sum is taken over all $y \in X \backslash\{x\}$ such that $G$ contains a $T_{x}-T_{y}$ edge. We shall prove that, for each of these $y$,

$$
\sum_{i \geqslant d} 2^{2 d-i}\left|A_{x, y}^{i}\right| \leqslant\left|V_{x}^{d}\right|
$$

so that (3) can be satisfied only if there are at least $2^{d+1}$ distinct $y$, ie. if $T_{x}$ sends edges to at least $2^{d+1}$ other trees $T_{y}$. Contracting all the trees $T_{x}$ with $x \in X$ we then obtain a minor of $G$ of minimum degree at least $2^{d+1}>k$, as desired.

For the proof of (4) let now $x$ and $y$ be fixed distinct vertices in $X$. Consider a $T_{x}-T_{y}$ edge $e=v w$ of $G$, with $v \in T_{x}$ and $w \in T_{y}$ say. Then $i:=d(v, x) \geqslant d$, by (1) and $w \notin T_{x}$. Let $z_{e}$ be the vertex below $v$ in $T_{x}$ at distance $d$ from $v$, ie. in $V_{x}^{i-d}$, and let $B_{e}$ be the set of vertices in $V_{x}^{d}$ that lie above $z_{e}$ in $T_{x}$. These vertices have distance $2 d-i$ from $z_{e}$, so

Let us show that

$$
\left|B_{e}\right| \geqslant 2^{2 d-i}
$$

$$
B_{e} \cap B_{e^{\prime}}=\emptyset \text { for all distinct } T_{x}-T_{y} \text { edges } e, e^{\prime} \text {. }
$$

Suppose not, ie. suppose that $z_{e}$ and $z_{e^{\prime}}$ are comparable in $T_{x}$, say $z_{e} \leqslant z_{e^{\prime}}$. Write $e=: v w$ and $e^{\prime}=: v^{\prime} w^{\prime}$ with $v, v^{\prime} \in T_{x}$ and $w, w^{\prime} \in T_{y}$, and put $i:=d(v, x)$. We show that the unique cycle $C$ in $T_{x} \cup T_{y}+e+e^{\prime}$ has length less than $g(G)$ (Fig. 1).

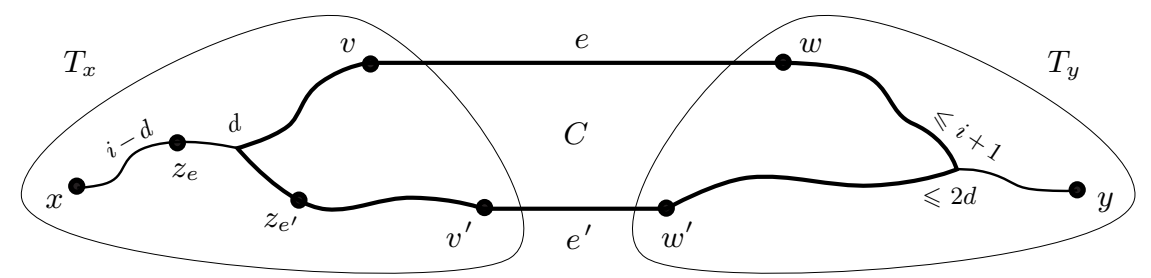

Figure 1. The cycle $C$ between $T_{x}$ and $T_{y}$.

The portion of $C$ in $T_{x}$ is a subpath of the walk $v \ldots z_{e} \ldots v^{\prime}$ in $T_{x}$, which has length at most $d+(2 d-(i-d))=4 d-i$. Its portion in $T_{y}$ is a subpath of the walk $w \ldots y \ldots w^{\prime}$ in $T_{y}$, which has length at most $(i+1)+2 d$ by $(2)$. Thus $|C| \leqslant 6 d+3<g(G)$, as desired. This completes the proof of (6).

Now (5), (6) and the definition of the $B_{e}$ imply (4):

$$
\sum_{i \geqslant d} 2^{2 d-i}\left|A_{x, y}^{i}\right|=\sum_{i \geqslant d} \sum_{e \in A_{x, y}^{i}} 2^{2 d-i} \underset{(5)}{\leqslant} \sum_{i \geqslant d} \sum_{e \in A_{x, y}^{i}}\left|B_{e}\right| \underset{(6)}{\leqslant}\left|V_{x}^{d}\right| .
$$


In order to improve the bound in Theorem 1 further, we have considered the question of whether the set $X$ might be chosen more effectively. For the proof of (1) we need its points to be more than $2 d$ apart. But if they were placed in $G$ so that every other vertex $v$ had distance $d(v, X) \leqslant \alpha d$ from $X$ for some $\alpha<2$ (rather than just $d(v, X) \leqslant 2 d$, which we get simply by choosing $X$ maximal), we would instantly shorten the cycle $C$ in the proof of (6) to at most $(2+2 \alpha) d+3$, improving the girth bound in the theorem to $(2+2 \alpha) \log k+3$. Note that the theoretical optimum of $\alpha=1$ would give us exactly (up to the additive constant) the conjectured lower bound from Section 2 .

The problem of whether such a set $X$ exists for given values of $d$ and $\alpha$ has been shown to be NP-hard [8], and so we did not pursue this approach further. However, Kühn and Osthus [6] have recently shown that a random choice of $X$ can indeed reduce the leading factor of 6 in Theorem 1 to the conjectured optimum of 4 .

\section{References}

[1] N. Biggs, Constructions for cubic graphs with large girth, Electronic J. Comb 5 (1998), Article A1 (25 pp).

[2] N. Biggs, \& M.J. Hoare, The sextet construction for cubic graphs, Combinatorica 3 (1983), 153-165.

[3] B. Bollobás, Extremal graph theory, Academic Press 1978.

[4] R. Diestel, Graph theory, 2nd edition, Springer-Verlag 2000 and http://www . math. uni-hamburg.de/home/diestel/books/graph. theory/download.html

[5] A.V. Kostochka, Lower bound of the Hadwiger number of graphs by their average degree, Combinatorica 4 (1984), 307-316.

[6] D. Kühn \& D. Osthus, Minors in graphs of large girth, Random Struct. Alg. 22 (2003), 213-225.

[7] W. Mader, Topological subgraphs in graphs of large girth, Combinatorica 18 (1998), 405-412.

[8] C. Rempel, Erzwingung von Teilstrukturen in Graphen durch globale Parameter, PhD Thesis, Hamburg 2001.

[9] C. Thomassen, Girth in graphs, J. Combin. Theory B 35 (1983), 129-141.

[10] A.G. Thomason, An extremal function for contractions of graphs, Math. Proc. Camb. Phil. Soc. 95 (1984), 261-265.

[11] A.G. Thomason, The extremal function for complete minors, J. Combin. Theory B $\mathbf{8 1}$ (2001) 318-338.

[12] A. Weiss, Girths of bipartite sextet graphs, Combinatorica 4 (1984), 241-245.

Mathematisches Seminar

Universität Hamburg

revised 15.2.2003

Bundesstraße 55

D - 20146 Hamburg

Germany 